\title{
Does oral vancomycin prophylaxis during systemic antibiotic exposure prevent Clostridioides difficile infection relapses? Still in search of an answer
}

\author{
Jeannie D. Chan PharmD, MPH ${ }^{1,2,3}$, Chloe Bryson-Cahn MD³, Rupali Jain PharmD ${ }^{2,3,4}$, John B. Lynch MD, MPH ${ }^{3}$ and \\ Catherine Liu MD 3 \\ ${ }^{1}$ Department of Pharmacy, Harborview Medical Center, Seattle, Washington, ${ }^{2}$ School of Pharmacy, University of Washington, Seattle, Washington, ${ }^{3}$ Department \\ of Medicine, Division of Allergy and Infectious Diseases, School of Medicine, University of Washington, Seattle, Washington and ${ }^{4}$ Department of Pharmacy, \\ University of Washington Medical Center, Seattle, Washington
}

To the Editor-Clostridioides difficile infection (CDI) represents a significant healthcare challenge, with $10 \%-30 \%$ of patients developing at least 1 recurrent episode, and the risk of relapses increases with each subsequent recurrence. ${ }^{1}$ Recurrent CDI is defined by resolution of CDI symptoms while on treatment, followed by symptom reappearance within 2-8 weeks after treatment completion. ${ }^{1}$ Exposure to systemic antibiotics in patients with prior CDI has been identified as an independent risk factor associated with CDI recurrence. ${ }^{1}$ Despite the lack of robust supporting evidence, secondary prophylaxis with oral vancomycin in patients on systemic antibiotics for infections other than CDI has become common practice. ${ }^{2-4} \mathrm{We}$ read "Oral vancomycin prophylaxis (OVP) during systemic antibiotic exposure to prevent Clostridioides difficile infection relapses" by Caroff et $\mathrm{al}^{4}$ with great interest. The authors reported that OVP was effective in reducing CDI relapse in patients with incident CDI ( 1 previous CDI) but not recurrent CDI ( $\geq 2$ CDI episodes).

We applaud the authors for conducting this study and taking into account potential confounders that were not adequately addressed in previous observational studies. ${ }^{2,3}$ In a small singlecenter study $(\mathrm{n}=203)$, Van Hise et $\mathrm{al}^{2}$ reported that OVP decreased the risk of CDI relapse (odds ratio, 0.12; 95\% CI, 0.04-0.4), though they did not differentiate between incident and recurrent CDI with multivariate analysis. Carignan et $\mathrm{al}^{3} \mathrm{dem}$ onstrated that the benefit of OVP in preventing CDI relapse was confined to patients with recurrent CDI. Importantly, the significant differences between the Carignan and Caroff investigations make it difficult to draw definitive conclusions, even in these larger-scale multicenter studies (Table 1).

In the Caroff study, the inclusion of hospitalized patients within 150 days of a positive $C$. difficile test did not capture ambulatory CDI cases, which may have explained the reported relapse rate of $9.5 \%$, which is substantially lower than the historical epidemiology of CDI. ${ }^{1}$ This low recurrence rate also raises questions about the circulating $C$. difficile strain type. NAP1 (North American pulsed-field gel electrophoresis type-1) strains are often a predictor of disease severity, recurrence, and increased mortality. ${ }^{1}$ The CDI relapse rate of $32.9 \%$ reported by Carignan et al may have overlapped with the NAP1 outbreak in Quebec, Canada, in $2002^{1}$

\footnotetext{
Author for correspondence: Jeannie D. Chan, PharmD, MPH, Harborview Medical Center, 325 Ninth Avenue, Box 359885, Seattle, WA 98104. E-mail: jdchan@uw.edu

Cite this article: Chan JD, et al. (2019). Does oral vancomycin prophylaxis during systemic antibiotic exposure prevent Clostridioides difficile infection relapses? Still in search of an answer. Infection Control \& Hospital Epidemiology, 40: 1084-1086, https:// doi.org/10.1017/ice.2019.192
}

and potentially with NAP1 strains still circulating in the community during their study periods between 2003 to 2011 . Because PCR ribotyping was not available in the OVP studies, it is unclear whether NAP1 strain may have contributed to the observed differences in relapse rates. The study populations are somewhat different as well: $50 \%$ of the patients were $>75$ years old in the Carignan study, whereas the average age in the Caroff study was 59-64 years old. In addition to older age being a risk factor for CDI recurrence, older adults may have impaired host defense because neutrophils are less able to phagocytize and kill C. difficile. ${ }^{3}$ This difference may partially indicate that host factors and humoral immunity play a role in CDI relapses.

Risk stratification of antibiotic class and duration of systemic antibiotic therapy should also be considered when calculating the risk of CDI recurrence given evidence suggesting that cumulative antibiotic exposures (ie, antibiotic dose, number of antibiotics, and days of antibiotic exposure) all appear to be associated with CDI risk. ${ }^{5}$ Caroff et al classified antibiotic risk as high, medium, and low, but the median duration of systemic antibiotic exposure was substantially shorter ( 6 days vs 14 days) than in the Carignan study. This difference led us to question whether a minimum threshold of antibiotic exposures is needed to derive benefit from OVP.

Because oral vancomycin may have deleterious effects to the indigenous microbiota of the colon and may promote colonization with vancomycin resistant Enterococci (VRE), carbapenem-resistant Klebsiella pneumoniae (KPC), and E. coli, it is imperative to carefully weigh the risks and benefits to determine the optimal dose and duration of OVP. ${ }^{6}$ The duration of OVP was much shorter (2.3 days vs 7 days) in the Caroff study, and vancomycin dose was not specified. The optimal dose of OVP has yet to be defined, and various dosing regimens for CDI may be used, for example $125 \mathrm{mg} 4$ times daily in the Carignan's study and 125-250 mg twice daily in the Van Hise study. With the increasing recognition of vancomycin's effect on the host microbiome, it seems prudent to define the lowest effective dose of OVP to minimize collateral damage. Because oral vancomycin is not systemically absorbed, high colonic vancomycin levels (500$1000 \mu \mathrm{g} / \mathrm{mL}$ ) can be achieved with $125 \mathrm{mg} 4$ times daily, which is several hundred-fold higher than the vancomycin minimum inhibitory concentration $\left(\mathrm{MIC}_{90}\right)$ for $\mathrm{C}$. difficile, which typically ranges between 1 and $2 \mu \mathrm{g} / \mathrm{mL}^{7,8}$ Vancomycin $125 \mathrm{mg}$ once daily may be sufficient as secondary prophylaxis for CDI. The duration of OVP is somewhat controversial in light of evidence suggesting that re-establishment of $C$. difficile colonization can occur within a 
Table 1. Comparison of Oral Vancomycin Prophylaxis (OVP) During Systemic Antibiotic Exposure in Preventing CDI Relapses

\begin{tabular}{|c|c|c|c|c|}
\hline Variable & \multicolumn{2}{|c|}{ Carignan et al } & \multicolumn{2}{|c|}{ Caroff et al } \\
\hline Study setting & \multicolumn{2}{|c|}{2 hospitals in Quebec, Canada } & \multicolumn{2}{|c|}{2 hospitals in Massachusetts, United States } \\
\hline Study period & \multicolumn{2}{|l|}{ 2003-2011 } & \multicolumn{2}{|l|}{ 2009-2015 } \\
\hline Inclusion criteria & \multicolumn{2}{|c|}{$\begin{array}{l}\geq 18 \text { yo with previous } C D I \text { requiring systemic } \\
\text { antibiotics within } 90 \mathrm{~d} \text { after } \mathrm{CDI} \text { diagnosis during } \\
\text { a hospital admission or a visit to a hospital } \\
\text { outpatient clinic. }\end{array}$} & \multicolumn{2}{|c|}{$\begin{array}{l}\text { Hospitalized patients requiring systemic } \\
\text { antibiotics within } 150 \mathrm{~d} \text { after a positive } C \text {. difficile } \\
\text { test }\end{array}$} \\
\hline Primary end point & \multicolumn{2}{|c|}{$\begin{array}{l}\text { CDI recurrence defined as diarrhea with positive } \\
\text { C. difficile toxin by toxin assay, enzyme-linked } \\
\text { immunoassay, PCR or colitis within } 90 \mathrm{~d} \text { after } \\
\text { systemic antibiotic exposure }\end{array}$} & \multicolumn{2}{|c|}{$\begin{array}{l}\text { CDI recurrence defined by either positive } C \text {. } \\
\text { difficile toxin assay or NAAT within } 90 \mathrm{~d} \text { after } \\
\text { systemic antibiotic exposure }\end{array}$} \\
\hline Study population & \multicolumn{2}{|c|}{$\begin{array}{l}551 \text { patients } \\
370 \text { initial } C D I, 172 \text { recurrent CDI } \\
\text { OVP, } 227(41 \%) \\
\text { No OVP, } 324(59 \%) \\
\text { Age, } 50 \% \text { with } \geq 75 \text { yo }\end{array}$} & \multicolumn{2}{|c|}{$\begin{array}{l}760 \text { patients } \\
474 \text { initial CDI, } 246 \text { recurrent CDI } \\
\text { OVP, } 193(25 \%) \text {; avg age: } 64 \text { yo } \\
\text { No OVP, } 567(75 \%) \text {; avg age: } 59 y \text { yo }\end{array}$} \\
\hline CDI relapse & \multicolumn{2}{|l|}{$32.9 \%$} & \multicolumn{2}{|l|}{$9.5 \%$} \\
\hline Median antibiotic exposure & \multicolumn{2}{|l|}{$14 \mathrm{~d}(\mathrm{IQR}, 10-17 \mathrm{~d})$} & \multicolumn{2}{|l|}{$\begin{array}{l}\text { OVP: } 5.99 \pm 6.29 \mathrm{~d} \\
\text { No OVP: } 5.41 \pm 6.65 \mathrm{~d}\end{array}$} \\
\hline Concomitant antibiotics & \multicolumn{2}{|c|}{$\begin{array}{l}\text { Fluoroquinolones (53\%), Carboxy/ureidopenicillins } \\
(25 \%)\end{array}$} & \multicolumn{2}{|c|}{$\begin{array}{l}71 \% \text { received high-risk antibiotics } \\
\text { (fluoroquinolones, cephalosporins, or } \\
\text { carbapenems) }\end{array}$} \\
\hline Median duration of OVP & \multicolumn{2}{|l|}{$7 \mathrm{~d}(\mathrm{IQR}, 3-12 \mathrm{~d})$} & \multicolumn{2}{|l|}{$2.3 \pm 5.8 d$} \\
\hline OVP dose & \multicolumn{2}{|c|}{$84 \%$ received $125 \mathrm{mg} 4$ times daily } & \multicolumn{2}{|l|}{ Dose not specified } \\
\hline Incident CDI & OVP $(n=137)$ & No OVP $(n=242)$ & OVP $(n=118)$ & No OVP $(n=353)$ \\
\hline $\mathrm{CDI}$ relapse $(\%)$ & $28(20.4)$ & $47(19.4)$ & $10(8.5)$ & $37(10.5)$ \\
\hline Adjusted ratio $(95 \% \mathrm{Cl})$ & aHR, $0.91(0.57-1.45)$ & Referent & aOR, $0.42(0.19-0.93)$ & Referent \\
\hline Recurrent CDI & OVP $(n=90)$ & No OVP $(n=82)$ & OVP $(n=64)$ & No OVP $(n=166)$ \\
\hline CDI relapse $(\%)$ & $49(54.4)$ & $57(69.5)$ & $7(10.9)$ & $13(7.8)$ \\
\hline Adjusted ratio $(95 \% \mathrm{Cl})$ & aHR, $0.47(0.32-0.69)$ & Referent & aOR, $1.19(0.42-3.33)$ & Referent \\
\hline
\end{tabular}

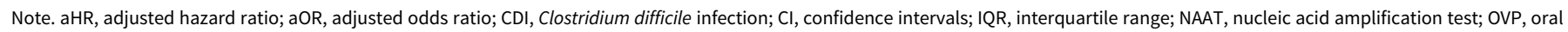
vancomycin prophylaxis; PCR, polymerase chain reaction.

few days to 3 weeks after CDI treatment. ${ }^{9}$ Should OVP be stopped at the course completion of systemic antibiotic or be extended to beyond the end of systemic antibiotic, and for how long?

Many unanswered questions remain, and the interpretations of these findings are limited by the inherent nature of retrospective observational studies and conflicting results. The heightened risk period for CDI recurrence is typically within 8 weeks after CDI treatment completion; ${ }^{1}$ it is unclear whether case ascertainment between 90 days versus 150 days from a prior CDI contributed to the inconsistent results between these 2 studies. Furthermore, the primary end points of CDI recurrence in existing observational studies are often defined only by positive $C$. difficile testing with or without diarrhea, making it difficult to distinguish between colonization and actual infection in the absence of clinical assessment. Prospective data are needed to guide the selection criteria of the targeted population with the highest propensity for CDI recurrence who may benefit most from the optimal dose and duration of OVP. We await results from ongoing randomized controlled trials to further inform the clinical utility of OVP as a preventive strategy in reducing the risk of recurrent CDI. ${ }^{10,11}$

Acknowledgments. None.
Financial support. No financial support was provided relevant to this article.

Conflicts of interest. All authors report no conflicts of interest relevant to this article.

\section{References}

1. McDonald LC, Gerding DN, Johnson S, et al. Clinical practice guidelines for Clostridium difficile infection in adults and children: 2017 update by the Infectious Diseases Society of America (IDSA) and Society for Healthcare Epidemiology of America (SHEA). Clin Infect 2018;66: 987-994.

2. Van Hise NW, Bryant AM, Hennessey EK, Crannage AJ, Khoury JA, Manian FA. Efficacy of oral vancomycin in preventing recurrent Clostridium difficile infection in patients treated with systemic antimicrobial agents. Clin Infect Dis 2016;63:651-653.

3. Carignan A, Poulin S, Martin P, et al. Efficacy of secondary prophylaxis with vancomycin for preventing recurrent Clostridium difficile infections. Am J Gastroenterol 2016;111:1834-1840.

4. Caroff DA, Menchaca JT, Zhang Zilu, et al. Oral vancomycin prophylaxis during systemic antibiotic exposure to prevent Clostridiodes difficile infection relapses. Infect Control Hosp Epidemiol 2019;40:662-667.

5. Stevens V, Dumyati G, Fine LS, Fisher SG, van Wijngaarden E. Cumulative antibiotic exposures over time and the risk of Clostridium difficile infection. Clin Infect Dis 2011;53:42-48. 
6. Lewis BB, Buffie CG, Carter RA, et al. Loss of microbiota-mediated colonization resistance to Clostridium difficile infection with oral vancomycin compared to metronidazole. J Infect Dis 2015;212:1656-1665.

7. Barlett JG. The case for vancomycin as the preferred drug for treatment of Clostridium difficile infection. Clin Infect Dis 2008;46:1489-1492.

8. Pepin J. Vancomycin for the treatment of Clostridium difficile infection: for whom is this expense bullet really magic? Clin Infect Dis 2008:46: $1493-1498$.
9. Abujamel T, Cadnum JL, Jury LA, et al. Defining the vulnerable period for re-establishment of Clostridium difficile colonization after treatment of $C$. difficile infection with oral vancomycin or metronidazole. PLoS One 2013;8(10):e76269.

10. Oral vancomycin to prevent recurrent Clostridium difficile infection with antibiotics. ClinicalTrials.gov Identifier: NCT03466502.

11. Efficacy of oral vancomycin prophylaxis for prevention of recurrent Clostridium difficile infection. ClinicalTrials.gov Identifier: NCT03462459.

\section{Increased time spent on terminal cleaning of patient rooms may not improve disinfection of high-touch surfaces}

\author{
Edmond A. Hooker MD, DrPH ${ }^{1,2}$ (1] \\ Xavier University, Cincinnati, Ohio and University of Cincinnati, Cincinnati, Ohio
}

To the Editor - I read with great interest the study by Coppin et al, ${ }^{1}$ in which they attempted to determine whether increased cleaning time would improve disinfection of high-touch surfaces. ${ }^{1}$ Although I applaud the attempt to address the issue, I am concerned about the very limited description of their methods. I also feel that the methodological flaws limit any conclusions from their research.

First, the authors allowed for the use of 3 very different disinfectants. They did not describe how each was utilized. Also, they did not describe what the EVS staff did for the increased time. If the environmental services staff (EVS) was not cleaning first and then disinfecting, it is not surprising that there was limited effect. Doing something wrong for longer does not improve cleaning. Also, previous research that showed EVS was much less effective at disinfection when they were not monitored. ${ }^{2}$ They only cultured 5 surfaces, and they did not culture the mattress, which is the highest touch point for the patient.

The bed manufacturers, in their revised instructions for use, require 5-6 steps to be performed when terminally cleaning a bed. These steps, if done properly, require 35 minutes to 1 hour to complete.

The authors also did not report how they cultured for bacteria. This could have made a huge difference in the results of the study. There is no description of the actual colony-forming units of bacteria found in each group before and after cleaning. The authors should have reported the log reduction in bacteria for each group, and statistical significance should have been reported as well.
The authors instead report predicted counts, which makes interpretation almost impossible.

I am concerned that this research will send the wrong message to hospitals: indicating that better cleaning will not work. It is extremely concerning that this research was funded by a manufacturer of an ultraviolet light disinfection machine. I strongly believe that cleaning better will not only decrease bacterial counts, but it will also decrease infections.

Author ORCIDs. Edmond A. Hooker, (D) https://orcid.org/0000-0001-73079483

Financial support. No financial support was provided relevant to this article.

Conflicts of interest. All authors report no conflicts of interest relevant to this article.

\section{References}

1. Coppin JD, Villamaria FC, Williams MD, Copeland LA, Zeber JE, Jinadatha C. Increased time spent on terminal cleaning of patient rooms may not improve disinfection of high-touch surfaces. Infect Control Hosp Epidemiol 2019:1-2.

2. Korchinski K, Hinkle C, Sopirala M. Effect of automated ultraviolet-C emitting device on disinfection of hospital rooms with and without real-time auditing of cleaning process. In: Open Forum Infectious Diseases, Vol. 3. New York: Oxford University Press; 2016.
Author for correspondence: Edmond Hooker, MD, DrPH, 3800 Victory Parkway, ML 5141, Cincinnati, OH 45207-5141. E-mail: hookere@xavier.edu

Cite this article: Hooker EA. (2019). Increased time spent on terminal cleaning of patient rooms may not improve disinfection of high-touch surfaces. Infection Control \& Hospital Epidemiology, 40: 1086, https://doi.org/10.1017/ice.2019.181 